\title{
Exact Partition Functions for the $q$-State Potts Model with a Generalized Magnetic Field on Lattice Strip Graphs
}

\author{
Shu-Chiuan Chang ${ }^{a, b} *$ and Robert Shrock ${ }^{b}$ \\ (a) Department of Physics, National Cheng Kung University, Tainan 70101, Taiwan and \\ (b) C. N. Yang Institute for Theoretical Physics and Department of Physics and Astronomy \\ Stony Brook University, Stony Brook, NY 11794, USA
}

\begin{abstract}
We calculate the partition function of the $q$-state Potts model on arbitrary-length cyclic ladder graphs of the square and triangular lattices, with a generalized external magnetic field that favors or disfavors a subset of spin values $\{1, \ldots, s\}$ with $s \leq q$. For the case of antiferromagnet spin-spin coupling, these provide exactly solved models that exhibit an onset of frustration and competing interactions in the context of a novel type of tensor-product $S_{s} \otimes S_{q-s}$ global symmetry, where $S_{s}$ is the permutation group on $s$ objects.
\end{abstract}

\section{INTRODUCTION}

We consider the $q$-state Potts model in a generalized external magnetic field that favors or disfavors a certain subset of spin values in the interval $I_{s}=\{1, \ldots, s\}$ with $s \leq q$. We present exact solutions for the partition function of this model on ladder strips of the square and triangular lattices with arbitrary length and periodic (i.e., cyclic) boundary conditions in the longitudinal $(x)$ direction and free boundary conditions in the transverse $(y)$ direction. This work extends our previous calculations of the partition function for the cyclic ladder graph in the zero-field case in Refs. [1, 2] and for the case of nonzero field and $s=1$ in Ref. [3].

In general, we denote a graph $G=(V, E)$ by its vertex set $V$ and its edge ( = bond) set $E$. The numbers of vertices, edges, and connected components of $G$ are denoted, respectively, by $n(G) \equiv n, e(G)$, and $k(G)$. We note that a triangular-lattice ladder graph of a given length may be obtained from the square-lattice ladder graph by adding a diagonal edge in each square, connecting, say, the lower left vertex to the upper right vertex in the square.

\footnotetext{
* (a): Permanent address; (b) Address on sabbatical
} 
In thermal equilibrium at temperature $T$, the partition function for the $q$-state Potts model on the graph $G$ in this generalized external magnetic field is given by

$$
Z=\sum_{\left\{\sigma_{i}\right\}} e^{-\beta \mathcal{H}},
$$

with the Hamiltonian

$$
\mathcal{H}=-J \sum_{\langle i j\rangle} \delta_{\sigma_{i}, \sigma_{j}}-\sum_{p=1}^{q} H_{p} \sum_{i} \delta_{\sigma_{i}, p},
$$

where $i$ and $j$ label vertices of $G, \sigma_{i}$ are spin variables with values in the set $I_{q}=\{1, \ldots, q\}$, $\beta=\left(k_{B} T\right)^{-1},\langle i j\rangle$ denote pairs of adjacent vertices, and $J$ is the spin-spin interaction constant. We denote the subset of the $q$ spin values orthogonal to $I_{s}$ as $I_{s}^{\perp}=\{s+1, \ldots, q\}$. The generalized magnetic field is defined as

$$
H_{p}=\left\{\begin{array}{ll}
H & \text { for } p \in I_{s} \\
0 & \text { for } p \in I_{s}^{\perp}
\end{array} .\right.
$$

Hence, for $H>0$ (respectively $H<0$ ), this model favors (resp. disfavors) spin values in the interval $I_{s}$. As is evident in Eqs. (1.1) and (1.2), the spin-spin interaction constant $J$ and the external magnetic field $H$ always appear in $Z$ multiplied by $\beta$, and, furthermore, in exponentiated form, so it is natural to define the following quantities:

$$
K=\beta J, \quad h=\beta H, \quad y=e^{K}, \quad v=y-1, \quad w=e^{h}
$$

The physical ranges of $v$ are $v \geq 0$ for the Potts ferromagnet, and $-1 \leq v \leq 0$ for the Potts antiferromagnet.

There are several motivations for this work. One motivation is the insight that it gives into how a new type of global symmetry is manifested in the structure of the partition function. If and only if $H=0$, then the zero-field Potts model Hamiltonian $\mathcal{H}$ and partition function $Z$ are invariant under the global symmetry group in which $\sigma_{i} \rightarrow g \sigma_{i} \forall i \in V$, with $g \in S_{q}$, where $S_{q}$ is the symmetric (= permutation) group on $q$ objects. We have used this symmetry property to choose, without loss of generality, the set of spins $I_{s}$ to be contiguous. Turning on a conventional external magnetic field that favors (or disfavors) one value of the $\sigma_{i}$ has the effect of simply reducing the original $S_{q}$ permutation symmetry group to $S_{q-1}$. (In the $q=2$ Ising case, this removes any global symmetry, since $S_{1}$ is trivial.) In contrast, with the generalized external magnetic field here, setting $H \neq 0$, reduces the original $S_{q}$ global permutation symmetry to the novel type of tensor-product global symmetry group

$$
\mathcal{G}_{\text {sym }}=S_{s} \otimes S_{q-s}
$$


This tensor-product symmetry group in the presence of $H \neq 0$ has interesting consequences for the structure of the partition function, as will be evident from several identities to be discussed below.

A second motivation for the present work is the connection with certain polynomials that appear in mathematical graph theory. In the case of zero external magnetic field, the partition function of the $q$-state Potts model is equivalent to a function of central interest in modern mathematical graph theory, namely the Tutte polynomial. If the spin-spin exchange constant, $J$, is negative, i.e., antiferromagnetic, the spin-spin interaction favors spin configurations such that spins on adjacent vertices are different. In the limit of this Potts antiferromagnet in which the temperature $T \rightarrow 0$ (i.e., $v \rightarrow-1$ ), the only spin configurations that make a nonzero contribution to the partition function are those such that spins on adjacent vertices have different values. It follows that the partition function for this zero-temperature $q$-state Potts antiferromagnet is equal to the chromatic polynomial $P(G, q)$, which counts the number of ways of assigning $q$ colors to the vertices of the graph $G$ subject to the condition that the colors on adjacent vertices are different. Such a color assignment is called a proper $q$-coloring of (the vertices of) $G$. Our generalization to a nonzero external magnetic field that favors or disfavors a subset of spin values establishes a further connection with mathematical graph theory. This connection is embodied in the purely graph-theoretic expression that we presented (as Eq. (12.7) in [4]) for the partition function. The zero-temperature limit of this partition function in the antiferromagnetic case defined what we have called a weighted-set graph coloring problem. Although we will not pursue applications here, we have discussed these earlier [4]. For example, the weighted-set graph coloring problem could describe the assignment of $q$ frequencies to commercial radio broadcasting stations in an area such that adjacent stations must use different frequencies to avoid interference and (i) for $H>0$, the stations prefer frequences in a set $I_{s}$ since reception is better for these or (ii) for $H<0$, the stations prefer to avoid the frequencies in the set $I_{s}$ because of poorer reception.

A third motivation is that our solutions provide exactly solved examples that exhibit the presence or absence of frustration and competing interactions in a controlled, parameterdependent manner. To explain this, we note that for a graph $G$, the minimum number of colors necessary to carry out a proper $q$-coloring of (the vertices of) $G$ is the chromatic number of $G$, denoted $\chi(G)$. If the spin-spin exchange interaction, $J$, is negative, then the minimization of the internal energy favors spin configurations in which the spins on adjacent vertices have different values. We consider this situation, $J<0$, for the remainder of this paragraph. Now in the presence of a nonzero external magnetic field $H$ that is positive (respectively, negative), the minimization of the internal energy favors spin values that lie 
in the interval $I_{s}$ (resp. $I_{s}^{\perp}$ ). The question of whether or not either (or both) of these two different situations involves competing interactions and associated frustration depends on the value of $s$ or $(q-s)$ relative to the chromatic number $\chi(G)$ of $G$. In the rest of this paragraph, we assume that $s \neq q$ since if $s=q$, then the partition function can be expressed completely (see Eq. (2.11) below) in terms of the partition function of a zero-field model multiplied by an overall factor. We also assume that $s$ does not have the formal value $s=0$, since then the $H$ field has no effect (see Eq. (2.7) below). Now, if $H>0$ and $0<s<\chi(G)$ or if $H<0$ and $0 \leq q-s<\chi(G)$, then the application of the respective positive or negative $H$ involves competing interactions and causes frustration. The reason for this can be explained as follows. If $H>0$ and $s<\chi(G)$, then the restriction preferred by the magnetic field interaction, that the spins should take values in $I_{s}$, conflicts with the restriction preferred by the spin-spin interaction, that adjacent spins should be different, requiring that they take

on values in the set $\{1, \ldots, \chi(G)\}$, which is larger than the interval $I_{s}$. The same statement applies for negative $H$, with the replacement of $s$ by $q-s$ and thus $I_{s}$ by $I_{s}^{\perp}$. This frustration becomes especially strong as the temperature $T \rightarrow 0$, i.e., $K \rightarrow \infty$ and $|h| \rightarrow \infty$ and for the infinite strip it leads to a nonanalytic change in the properties of the ground state as a function of $H /|J|$ if $J<0$ and $s<\chi(G)$. A particularly useful aspect of the present work is that one can see the origin of this nonanalyticity explicitly from the structure of the exactly solved free energy. (For other examples of frustration and competing interactions in spin models, see, e.g., [5].)

More generally, an obvious motivation for exact calculations of partition functions of statistical mechanical models is the insights that they can give concerning the properties of these models that complement those obtained from numerical simulations and series expansions.

This paper is organized as follows. In Sect. [I] we review basic properties of $Z(G, q, s, v, w)$ for an arbitrary graph $G$. In Sect. III we recall some general structural results for $Z(G, q, s, v, w)$ where $G$ is an arbitrarily long cyclic strip graph of the square or triangular lattice that will be relevant for our specific calculations. In Sects. IV and $\mathrm{V}$ we present our calculations of this partition function for arbitrarily long ladder graphs of the square and triangular lattices, respectively. In Sect. VI we discuss some physical consequences of these calculations. Sect. VII contains our conclusions, and several relevant formulas are given in the Appendices A,C.

\section{BASIC PROPERTIES}

In this section we will briefly review some basic properties of the partition function that are relevant for the present work. Recall that a spanning subgraph $G^{\prime} \subset G$ is a graph consisting 
of the same set of vertices and a subset of the edges of $G$, i.e., $G^{\prime}=\left(V, E^{\prime}\right)$ with $E^{\prime} \subset E$. It is quite useful to have a formula that expresses $Z$ in a purely graph-theoretic manner, instead of as a sum over the spin configurations $\sigma_{i}$. This has been achieved by expressing $Z$ as a sum of contributions from the disjoint components $G_{i}$ comprising spanning subgraphs $G^{\prime} \subseteq G$. The simplest formulation of a $q$-state Potts model in an external magnetic field $H$ takes this field to favor or disfavor one spin value out of the $q$ possible values. In our notation, this is the case $s=1$, i.e., the interval $I_{s}$ consists of a single spin value which, without loss of generality, can be taken to be $\sigma_{i}=1$. For this $s=1$ case, a graph-theoretic formula for the partition function was given in [6] (reviewed in [7]). In Refs. [3] and [4, 8] we studied this case further and presented a number of properties of the resultant partition function for various families of graphs. As discussed in the introduction, a further generalization is to take the external magnetic field to favor or disfavor a set of several spin values in an interval $I_{s}$ rather than just a single value. For this $s>1$ case, we gave a general formula for the partition function for an arbitrary graph $G$ (as Eq. (12.7)) in Ref. [4]. Refs. [9, 10] derived a number of properties of this partition function and reported exact calculations of it for several families of graphs. Let us denote the number of connected components of $G$ as $k(G)$ and the connected subgraphs of a spanning subgraph $G^{\prime}$ as $G_{i}^{\prime}, i=1, . ., k\left(G^{\prime}\right)$. Our formula is [4]

$$
Z(G, q, s, v, w)=\sum_{G^{\prime} \subseteq G} v^{e\left(G^{\prime}\right)} \prod_{i=1}^{k\left(G^{\prime}\right)} u_{n\left(G_{i}^{\prime}\right)}
$$

where

$$
u_{m}=q-s+s w^{m}=q+s\left(w^{m}-1\right) .
$$

It follows from Eq. (2.1) that $Z$ is a polynomial in the variables $q, s, v$, and $w$, and this is indicated in the notation $Z(G, q, s, v, w)$. Note that Eqs. (2.1) and (2.2) also allow one to generalize $q$ and $s$ from the positive integers to the real numbers. (Indeed, studies of zeros of $Z(G, q, s, v, w)$ in $q$ and/or $s$ for fixed $v$ and $w$ require that one generalize $q$ and $s$ further to the complex numbers [4, 9, 10].) Without loss of generality, we restrict ourselves here to connected graphs $G$; however, the spanning subgraphs $G^{\prime}$ in Eq. (2.1) may contain (and most do contain) more than one connected component.

The case $v=-1$ yields a weighted-set chromatic polynomial, denoted $\operatorname{Ph}(G, q, s, w)$, that describes a graph coloring problem in which one assigns $q$ colors to the vertices of a graph such that adjacent vertices have different colors, with a vertex weighting $w$ that either disfavors or favors a given subset of $s$ colors contained in the set of $q$ colors [8]-[4]. In our notation,

$$
P h(G, q, s, w) \equiv Z(G, q, s,-1, w)
$$


As a quantity encoding information about a graph, the weighted-set chromatic polynomial $\operatorname{Ph}(G, q, s, w)$ is more powerful than the chromatic polynomial, since it can distinguish between graphs that are chromatically equivalent, i.e., are different but have the same chromatic polynomial [4]-[10].

The zero-field $q$-state Potts model with partition function $Z(G, q, v)$ is equivalent to a function of central importance in mathematical graph theory, the Tutte polynomial [11, 12] (early reviews include [13, 14])

$$
Z(G, q, v)=(x-1)^{k(G)}(y-1)^{n(G)} \sum_{G^{\prime} \subseteq G}(x-1)^{k\left(G^{\prime}\right)-k(G)}(y-1)^{c\left(G^{\prime}\right)},
$$

where

$$
x=1+\frac{q}{v}
$$

and $y$ was given in Eq. (1.4), so that $q=(x-1)(y-1)$. In Eq. (2.4), $c\left(G^{\prime}\right)$ denotes the number of linearly independent circuits (cycles) in $G$, satisfying $c\left(G^{\prime}\right)=e\left(G^{\prime}\right)+k\left(G^{\prime}\right)-n\left(G^{\prime}\right)$. Just as $\operatorname{Ph}(G, q, s, w)$ generalizes the chromatic polynomial, so also $Z(G, q, s, v, w)$ generalizes the Tutte polynomial. For example, $Z(G, q, s, v, w)$ can distinguish between different graphs with the same Tutte polynomnial [9, 10].

We next review some basic identities and symmetry properties of $Z(G, q, s, v, w)$. From the relation $w^{m}-1=(w-1) \sum_{j=0}^{m-1} w^{j}$ with $m=n\left(G_{i}^{\prime}\right)$ in conjunction with Eqs. (2.1) and (2.2), it follows that the variable $s$ enters in $Z(G, q, s, v, w)$, and $\operatorname{Ph}(G, q, s, w)$ only in the combination $s(w-1)$. Furthermore, $Z(G, q, s, v, w)$ satisfies the following identities. First, if $h=0$, i.e., $w=1$, then the partition function reduces to the zero-field expression result, i.e.,

$$
Z(G, q, s, v, 1)=Z(G, q, v) .
$$

An equivalent result is obtained if one formally sets $s=0$, i.e., the set of spin values that interact with the external field is the null set:

$$
Z(G, q, 0, v, w)=Z(G, q, v) .
$$

The equivalence of Eqs. (2.6) and (2.7) is in accord with the above-mentioned property that $Z(G, q, s, v, w)$ depends on the variables $s$ and $w$ only via the combination $s(w-1)$, so $w=1$ is equivalent to $s=0$. Since the right-hand sides of Eqs. (2.6) and (2.7) do not depend on $s$ or $w$, they also imply the partial differential equations

$$
\frac{\partial P h(G, q, s, 1)}{\partial s}=0
$$

and

$$
\frac{\partial P h(G, q, 0, w)}{\partial w}=0
$$


The condition $w=0$ means that spin configurations with $\sigma_{i} \in I_{s}$ for any $i$ make no contribution to the partition function, leading to the identity

$$
Z(G, q, s, v, 0)=Z(G, q-s, v)
$$

If $s=q$, then all of the spin values receive the same weighting due to the generalized external magnetic field, so that

$$
Z(G, q, q, v, w)=w^{n} Z(G, q, v) .
$$

Finally, from the global tensor-product symmetry (1.5) of the theory in the presence of the generalized magnetic field, there is an identity involving the interchange of the intervals $I_{s}$ and $I_{q-s}$ combined with a reversal in the sign of $h$, namely

$$
Z(G, q, s, v, w)=w^{n} Z\left(G, q, q-s, v, w^{-1}\right)
$$

Setting $v=-1$ in these identities (2.6)-(2.12) yields the corresponding relations for $\operatorname{Ph}(G, q, s, w)$; for example, $\operatorname{Ph}(G, q, s, w)=w^{n} P h\left(G, q, q-s, w^{-1}\right)$, etc.

\section{PARTITION FUNCTION FOR CYCLIC STRIP GRAPHS}

In this section we recall some general structural results from [9] that apply to $Z(G, q, s, v, w)$ for strip graphs of the square or triangular lattice, denoted generically as $\Lambda$, of arbitarily great length $L_{x}=m$ vertices and transverse width $L_{y}$ vertices with free transverse boundary conditions and periodic (cyclic) longitudinal boundary conditions. We denote a strip graph of this type as $G=\Lambda, L_{y} \times m, c y c$.. The partition function $Z(G, q, s, v, w)$ has the general form

$$
Z\left(\Lambda, L_{y} \times m, c y c ., q, s, v, w\right)=\sum_{d=0}^{L_{y}} \tilde{c}^{(d)}(q) \sum_{j=1}^{n_{Z h}\left(L_{y}, d, s\right)}\left[\lambda_{Z, \Lambda, L_{y}, d, j}(q, s, v, w)\right]^{m}
$$

where

$$
\tilde{c}^{(d)}(q) \equiv c^{(d)}(\tilde{q})=\sum_{j=0}^{d}(-1)^{j}\left(\begin{array}{c}
2 d-j \\
j
\end{array}\right) \tilde{q}^{d-j}
$$

and $\tilde{q}=q-s$. The first few $\tilde{c}^{(d)}(q)$ are $\tilde{c}^{(0)}(q)=1$,

$$
\tilde{c}^{(1)}(q)=\tilde{q}-1=q-s-1,
$$

and

$$
\tilde{c}^{(2)}(q)=\tilde{q}^{2}-3 \tilde{q}+1=q^{2}-2 q s+s^{2}+3(s-q)+1 .
$$


As is evident in the exact solution for the circuit graph in [9] and in our new solutions, certain $\lambda_{Z, \Lambda, L_{y}, d, j}$ terms occur with multiplicities greater than 1, which are polynomials in $s$. The numbers $n_{Z h}\left(L_{y}, d, s\right)$ were derived for $s=1$ (as Theorem 2.1 with Table 1) in [3], and results for arbitrary $s \in I_{s}$ were given in [9]. We have

$$
\begin{gathered}
n_{Z h}\left(L_{y}, L_{y}, s\right)=1, \\
n_{Z h}\left(L_{y}, L_{y}-1, s\right)=(s+1) L_{y}+\left(L_{y}-1\right), \\
n_{Z h}\left(L_{y}+1,0, s\right)=(s+1) n_{Z h}\left(L_{y}, 0, s\right)+n_{Z h}\left(L_{y}, 1, s\right),
\end{gathered}
$$

and, for $1 \leq d \leq L_{y}+1$,

$$
n_{Z h}\left(L_{y}+1, d, s\right)=n_{Z h}\left(L_{y}, d-1, s\right)+(s+2) n_{Z h}\left(L_{y}, d, s\right)+n_{Z h}\left(L_{y}, d+1, s\right) .
$$

For the present case $L_{y}=2$, these results yield $n_{Z h}(2,2, s)=1$ as a special case of (3.5) ,

$$
n_{Z h}(2,1, s)=2 s+3
$$

and

$$
n_{Z h}(2,0, s)=s^{2}+2 s+2 .
$$

The total number of $\lambda_{Z, s q, L_{y}, d, j}$ terms is

$$
N_{Z h, L_{y}, s}=\sum_{d=0}^{L_{y}} n_{Z h}\left(L_{y}, d, s\right) .
$$

If $s=1$, then [8]

$$
N_{Z h, L_{y}, 1}=\sum_{j=0}^{L_{y}}\left(\begin{array}{c}
L_{y} \\
j
\end{array}\right)\left(\begin{array}{c}
2 j \\
j
\end{array}\right)
$$

For general $s$ [9],

$$
N_{Z h, L_{y}, s}=\sum_{j=0}^{L_{y}}\left(\begin{array}{c}
L_{y} \\
j
\end{array}\right)\left(\begin{array}{c}
2 j \\
j
\end{array}\right) s^{L_{y}-j} .
$$

For general $s$ and $L_{y}=2$, Eq. (3.11) yields

$$
N_{Z h, 2, s}=\sum_{d=0}^{2} n_{Z h}(2, d, s)=s^{2}+4 s+6 .
$$

A general result is that the unique $\lambda_{Z, s q, L_{y}, d, j}$ for $d=L_{y}$ (for which we thus suppress the index $j$ ) is unique, i.e., has multiplicity 1 , and is given by

$$
\lambda_{Z, s q, L_{y}, L_{y}, j} \equiv \lambda_{Z, s q, L_{y}, L_{y}}=v^{L_{y}} .
$$


The $s=1$ special case of this was given (as Eq. (4.4)) in [3] .

The symmetry (2.12) under the replacement of $s$ by $q-s$ and $w$ by $w^{-1}$, namely $Z(G, q, s, v, w)=w^{n} Z\left(G, q, q-s, v, w^{-1}\right)$, has quite interesting consequences for the structure of this partition function on cyclic lattice strip graphs (of arbitrary length). A basic result is that under the replacement $s \rightarrow q-s$, a coefficient $\tilde{c}^{(d)}(q)=c^{(d)}(\tilde{q})$ changes to $c^{(d)}(s)$. Hence, taking into account that the $L_{y} \times m$ cyclic strip of the square or triangular lattice has $n=L_{x} L_{y}=m L_{y}$ vertices, the symmetry (2.12) is realized as

$$
\begin{aligned}
& Z\left(\Lambda, L_{y} \times m, c y c ., q, s, v, w\right)=w^{m L_{y}} Z\left(\Lambda, L_{y} \times m, c y c ., q, q-s, v, w^{-1}\right) \\
= & \sum_{d=0}^{L_{y}} c^{(d)}(s) \sum_{j=1}^{n_{Z h}\left(L_{y}, d, q-s\right)}\left[w^{L_{y}} \lambda_{Z, \Lambda, L_{y}, d, j}\left(q, q-s, v, w^{-1}\right)\right]^{m} .
\end{aligned}
$$

In order for this identity to hold, there must be an interplay between changes in four different quantities: (i) the numbers $n_{Z h}\left(L_{y}, d, s\right)$ in Eq. (3.1) which become $n_{Z h}\left(L_{y}, d, q-s\right)$ in Eq. (3.16); (ii) the related multiplicity factors for various $\lambda_{Z, \Lambda, L_{y}, d, j}$, which were polynomials in $s$ in Eq. (3.1) and become polynomials in $q-s$ in (3.16); the (iii) the coefficients $\tilde{c}^{(d)}(q)=$ $c^{(d)}(q-s)$ in (3.1), which become $c^{(d)}(s)$ in (3.16); and (iv) the terms $\left(\lambda_{Z, \Lambda, L_{y}, d, j}\right)^{m}$, which also change under the replacements $s \rightarrow q-s, w \rightarrow w^{-1}$, and multiplication by $w^{m L_{y}}$.

It is natural to analyze how this symmetry (2.12) is realized in the simplest case, namely for the circuit graph. Here, $n_{Z h}(1,0, s)=s+1$, and $n_{Z h}(1,1, s)=1$. The exact partition function for this graph, $Z(1 D ; 1 \times m, c y c ., q, s, v, w) \equiv Z\left(C_{m}, q, s, v, w\right)$ for the circuit graph was given in [9] and is

$$
Z\left(C_{m}, q, s, v, w\right)=\sum_{j=1}^{s+1}\left[\lambda_{Z, C, 0, j}(q, s, v, w)\right]^{m}+\tilde{c}^{(1)}(q) v^{m}
$$

where the $\lambda_{Z, C, d, j}$ terms are as follows. For $d=1$, there is a single term

$$
\lambda_{Z, C, 1,1}=v \equiv \lambda_{Z, C, 1}
$$

For $d=0$, the $\lambda_{Z, C, 0, j}$ consist of the two roots of a quadratic, denoted as $\lambda_{Z, C, 0, \pm}$,

$$
\lambda_{Z, C, 0, \pm}=\frac{1}{2}[A \pm \sqrt{R}]
$$

where

$$
\begin{gathered}
A=q+s(w-1)+v(w+1), \\
R=A^{2}-4 v(q+v) w,
\end{gathered}
$$


and, if $s \geq 2$, also the terms

$$
\lambda_{Z, C, 0, j}=v w \text { for } 3 \leq j \leq s+1
$$

That is, provided that $s \geq 2$, the terms $\lambda_{Z, C, 0, j}$ are all the same for $j=3, \ldots, s+1$ and hence can be considered to be a single $\lambda$ with multiplicity $s-1$. Hence, one can re-express $Z\left(C_{m}, q, s, v, w\right)$ in a different but equivalent form (given as Eq. (5.9) in [10]),

$$
Z\left(C_{m}, q, s, v, w\right)=\sum_{j=1}^{2}\left[\lambda_{Z, C, 0, j}\right]^{m}+(s-1)(v w)^{m}+(q-s-1) v^{m} .
$$

For this circuit graph, the symmetry (2.12) involves the combined effects of changes in four quantities: (i) the numbers $n_{Z h}(1,1, s)=1$ and $n_{Z h}(1,0, s)=s+1$, (ii) the multiplicity factors for the various terms $\lambda_{Z, \Lambda, L_{y}, d, j}$, in particular the multiplicity factor $s-1$ for a subset of the $\lambda_{Z, C, 0, j}$ terms; (iii) the coefficient $\tilde{c}^{(1)}(q)=q-s-1$; and (iv) the terms $\left(\lambda_{Z, C, d, j}\right)^{m}$ themselves. Since $\tilde{c}^{(0)}(q)=1$ and the quadratic roots $\lambda_{Z, C, 0, \pm}$ each occur with multiplicity 1 , it follows that they must transform into $w^{-1}$ times themselves, i.e.,

$$
s \rightarrow q-s \Rightarrow \lambda_{Z, C, 0, \pm} \rightarrow w^{-1} \lambda_{Z, C, 0, \pm}
$$

so that with the inclusion of the factor $w^{m}$ in the relevant $L_{y}=1$ case of Eq. (3.16), the $\left[\left(\lambda_{Z, C, 0,+}\right)^{m}+\left(\lambda_{Z, C, 0,-}\right)^{m}\right]$ in the $d=0$ sector is transformed into itself. The other part of the $d=0$ sector is comprised of the term $(s-1)(v w)^{m}$ coming from the $m$ 'th power of $\lambda_{Z, C, 0, j}=v w$ with multiplicity $s-1$. Under the replacements $s \rightarrow q-s$ and $w \rightarrow w^{-1}$, this term transforms into $(q-s-1)(v / w)^{m}$, and with inclusion of the $w^{m}$ prefactor, this becomes $(q-s-1) v^{m}$. Under the same replacements $s \rightarrow q-s$ and $w \rightarrow w^{-1}$, the $d=1$ term in $Z\left(C_{m}, q, s, v, w\right), \tilde{c}^{(1)}(q) v^{m}=(q-s-1) v^{m}$, transforms into $(s-1) v^{m}$, so with inclusion of the $w^{m}$ prefactor, this becomes $(s-1)(v w)^{m}$. Thus, under this replacement, the second and third terms in $Z(C, q, s, v, w)$ interchange with each other. This involves what could be called a "transmigration" of terms, in which terms from a sector with value of $d$ switch with terms in a sector with a different value of $d$.

It is also instructive to see how this exact solution satisfies the identity (2.6) for the special case of zero field, $h=0$, i.e., $w=1$. In this case, the $s-1 \lambda_{Z, C, 0, j}$ with $3 \leq j \leq s+1$ become equal to $\lambda_{Z, C, 1}=v$. In this $w=1$ special case the quadratic roots reduce to $q+v$ and $v$. Thus, $(s-1)+1=s$ terms $v^{m}$ associated with the coefficient $\tilde{c}^{(0)}(q)=1$ become equal to, and can be grouped with, the $v^{m}$ term associated with the coefficient $\tilde{c}^{(1)}(q)=q-s-1$, yielding the term $v^{m}$ with coefficient $s+(q-s-1)=q-1=c^{(1)}$. This shows how the zero-field form of the partition function emerges in this limit. In [4], in the context of the analogous 
phenomenon for the weighted-set chromatic polynomial $\operatorname{Ph}(G, q, s, w)$, we remarked on this and denoted it as a "transmigration" process, in which $\lambda \mathrm{s}$ in a subset of the $\lambda$ s associated with the coefficient $\tilde{c}^{(d)}(q)$ become equal to a $\lambda$ associated with a coefficient $\tilde{c}^{\left(d^{\prime}\right)}(q)$ with a different degree $d^{\prime}$, so that, taking account of their respective coefficients and multiplicities, they can be grouped together. The same phenomenon occurs, in a more complicated manner, for $Z(s q, 2, q, s, v, w)$ and $Z(\operatorname{tri}, 2, q, s, v, w)$.

\section{PARTITION FUNCTION FOR CYCLIC SQUARE-LATTICE LADDER GRAPHS}

Next, we present our exact results for the partition functions of the $q$-state Potts model in a generalized magnetic field on cyclic ladder graphs of the square lattices of length $L_{x}=$ $m$ vertices in the longitudinal direction and $L_{y}=2$ vertices in the transverse direction, $Z(s q, 2 \times m, c y c ., q, s, v, w)$. This graph has $n=2 L_{x}$ vertices. The partition function has the form of (3.1) with $n_{Z h}(2,2, s)=1$ as a special case of (3.5), and with the $n_{Z h}(2, d, s)$ given in Eq. (3.9) for $d=1$ and in Eq. (3.10) for $d=0$. As noted above, our new result generalizes the zero-field solution given in [1] and the solution for the special case $s=1$ given in [3].

We calculate the $\lambda_{Z, s q, 2, d, j} s$ to be the following:

- For $d=2$, as the $L_{y}=2$ special case of $(3.15)$, there is a single term

$$
\lambda_{Z, s q, 2,2,1} \equiv \lambda_{Z, s q, 2,2}=v^{2} \text {. }
$$

- For the $d=1$ sector, i.e., the $\lambda_{Z, s q, 2, d, j}$ s with coefficient $\tilde{c}^{(1)}(q)$, there are $2 s+3$ terms. We find the following results: (i) terms equal to $v^{2} w$, with multiplicity $2(s-1)$, which thus may be denoted as

$$
\lambda_{Z, s q, 2,1, j}=v^{2} w \quad \text { for } 1 \leq j \leq 2(s-1),
$$

(ii), next, the terms $\lambda_{Z, s q, 2,1, j}$ with $j=2 s-1$ and $j=2 s$, which we denote simply as $\lambda_{Z, s q, 2,1, \pm}$ :

$$
\lambda_{Z, s q, 2,1, \pm}=\frac{v}{2}[A \pm \sqrt{R}]
$$

satisfying

$$
\lambda_{Z, s q, 2,1, \pm}=v \lambda_{Z, C, 1,0, \pm}
$$

where $\lambda_{Z, C, 1,0, \pm}, A$, and $R$, were given in Eqs. (3.19)-(3.21), and finally, (iii) the terms $\lambda_{s q, 2,1, j}$ with $2 s+1 \leq j \leq 2 s+3$, which are the roots of the cubic equation

$$
\lambda^{3}-v[q+s(w-1)+v(v+4+w)] \lambda^{2}
$$




$$
\begin{aligned}
& +v^{3}[q(w+1)+s(w-1)(v+1)+v\{q+1+v(w+1)+4 w\}] \lambda \\
& -(q+v)(1+v) v^{5} w=0 .
\end{aligned}
$$

The $\lambda_{Z, s q, 2,1, \pm}$ terms in Eq. (4.3) and the three $\lambda_{Z, s q, 2,1, j}$ terms that are the roots of Eq. (4.5) each have multiplicity 1.

- For $d=0$, we have terms $\lambda_{Z, s q, 2,0, j}$ with $1 \leq j \leq s^{2}+2 s+2$. Since the ordering of the index $j$ is a convention, we do not specify it below. These terms and their multiplicities are

$$
(i): \quad v(q+v) w \text { with multiplicity } 1,
$$

$$
\text { (ii) : } \quad(v w)^{2} \text { with multiplicity } s^{2}-3 s+1,
$$

two quadratic roots, each with multiplicity $s-1$ : (iii)

$$
\lambda_{Z, s q, 2,0, \pm}=\frac{v w}{2}[A \pm \sqrt{R}]
$$

which satisfy

$$
\lambda_{Z, s q, 2,0, \pm}=w \lambda_{Z, s q, 2,1, \pm}=w v \lambda_{Z, C, 1,0, \pm}
$$

(iv) the three roots of the following cubic equation, each appearing with multiplicity $s-1$ :

$$
\begin{aligned}
& \lambda^{3}-v w\left[q+s(w-1)+v(1+4 w)+v^{2} w\right] \lambda^{2} \\
& +(v w)^{3}\left[2 q+s(w-1)(v+1)+v(q+4+w)+v^{2}(w+1)\right] \lambda \\
& -(v w)^{5}(q+v)(1+v)=0,
\end{aligned}
$$

and (v) the five roots of an algebraic equation of degree 5, each with multiplicity 1. This quintic equation is the characteristic polynomial of the transfer matrix $T_{Z, s q, 2,0}$ given in Appendix B,

In the special case $s=1$, Eqs. (3.9) and (3.10) yield $n_{Z h, 2,1}=5$ and $n_{Z h, 2,0}=5$, respectively. For this special case we have checked that the $\lambda_{Z, s q, 2,1, j}$ and $\lambda_{Z, s q, 2,0, j}$ for $j=$ $1, \ldots, 5$ agree, respectively, with the eigenvalues that we calculated of the transfer matrices $T_{Z, s q, 2,1}$ in Eq. (4.18) and $T_{Z, s q, 2,0}$ in Eq. (8.1) of [3].

It is useful to describe how these $\lambda_{Z, s q, 2, d, j}$ terms reduce to the known results for $Z(s q, 2 \times$ $m, q, v)$ in the zero-field case presented in [1] (listed, for reference, in Appendix [A). To 
distinguish the $\lambda_{s q, 2, d, j}$ terms in the zero-field partition function $Z(s q, 2 \times m, q, v)$ from the $\lambda_{Z, s q, 2, d, j}$ terms in the field-dependent partition function $Z(s q, 2 \times m, q, s, v, w)$, we suppress the subscript $Z$ in the former. Among the $\lambda_{Z, s q, 2, d, j}$ with $d=1$, first, the $\lambda_{Z, s q, 2,1, j}$ with $1 \leq j \leq 2(s-1)$ obviously reduce to $v^{2}=\lambda_{s q, 2,2}$. The quadratic roots $\lambda_{Z, s q, 1, j}$ with $j=2 s-1$ and $2 s$ reduce, respectively as

$$
\begin{gathered}
\lambda_{Z, s q, 2,1,+}=v(q+v) \quad \text { for } w=1, \\
\lambda_{Z, s q, 2,1,-}=v^{2} \quad \text { for } w=1 .
\end{gathered}
$$

The roots of the cubic equation (4.5) reduce to $v^{2}$ and to $\lambda_{s q, 2,1, \pm}$ in Eq. (A5), each with multiplicity 1 .

In the $d=0$ sector, the term (i) reduces to $v(q+v)$ with multiplicity 1 and (ii) reduces to $v^{2}$ with multiplicity $s^{2}-3 s+1$. The $\lambda_{Z, s q, 2,0, \pm}$ reduce to $v(q+v)$ and $v^{2}$, each with multiplicity $s-1$. The roots of the cubic equation (4.10) reduce to $v^{2}$ and $\lambda_{s q, 2,1, \pm}$ in Eq. (A5), each with multiplicity $s-1$. Finally, the roots of the quintic equation reduce to $v^{2}$, $\lambda_{s q, 2,1, \pm}$ in Eq. (A5), and $\lambda_{s q, 2,0, \pm}$ in Eq. (A6), each with multiplicity 1 . It is then readily confirmed that in this zero-field case $w=1$, our result satisfies the identity

$$
Z(s q, 2 \times m, c y c ., q, s, v, 1)=Z(s q, 2 \times m, c y c ., q, v)
$$

with $Z(s q, 2 \times m, c y c ., q, v)$ calculated in [1], as given in Eqs. (A3)-(A5). We have also confirmed that our result satisfies the various identities given in Sect. II and that in the special case $s=1$, it reduces to our previous solution of $Z(s q, 2 \times m, c y c ., q, 1, v, w)$ given in [3].

\section{PARTITION FUNCTION FOR CYCLIC TRIANGULAR-LATTICE LADDER GRAPHS}

In this section we present our exact results for the partition functions of the $q$-state Potts model in a generalized magnetic field on cyclic ladder graphs of the triangular lattice of length $L_{x}=m$ vertices in the longitudinal direction, $Z(\operatorname{tri}, 2 \times m, c y c ., q, s, v, w)$. As was the case with the square-lattice ladder strip, the partition function for the triangular-lattice ladder strip has the form of (3.1) with $n_{Z h}(2,2, s)=1$ as a special case of (3.5), and with the $n_{Z h}(2, d, s)$ given in Eq. (3.9) for $d=1$ and in Eq. (3.10) for $d=0$. Our result generalizes the zero-field solution given in [2] and the solution for the special case $s=1$ given in [3]

We calculate the $\lambda_{Z, t r i, 2, d, j} s$ to be as follows: 
- For $d=2$, there is a single term,

$$
\lambda_{Z, t r i, 2,2,1} \equiv \lambda_{Z, t r i, 2,2}=v^{2}
$$

as the $L_{y}=2$ special case of $(3.15)$;

- For the $d=1$ sector, i.e., the $\lambda_{Z, t r i, 2, d, j}$ s with coefficient $\tilde{c}^{(1)}(q)$, there are $2 s+3$ terms. We find the following results: (i) terms equal to $v^{2} w$, with multiplicity $2(s-1)$, which thus may be denoted as

$$
\lambda_{Z, t r i, 2,1, j}=\lambda_{Z, s q, 2,1, j}=v^{2} w \quad \text { for } 1 \leq j \leq 2(s-1)
$$

(ii) next, five terms $\lambda_{Z, t r i, 2,1, j}$ with $2 s-1 \leq j \leq 2 s+3$ which are the roots of a quintic equation that is the characteristic polynomial of the transfer matrix $T_{Z, t r i, 2,1}$ given in Appendix C.

- For $d=0$, there are $s^{2}+2 s+2$ terms $\lambda_{Z, t r i, 2,0, j}$. Of these, one set coincides with the corresponding set for the square-lattice strip, namely (i): $(v w)^{2}$, each occurring with multiplicity $s^{2}-3 s+1$. The remaining terms include (ii) five roots of a quintic equation which is the characteristic polynomial of the transfer matrix $T_{Z, t r i, 2,0 a}$ (see Appendix (C), each with multiplicity $s-1$, and (iii) six terms which are the roots of a degree- 6 equation that is the characteristic polynomial of the transfer matrix $T_{Z, t r i, 2,0 b}$ given in Appendix C, each with multiplicity 1.

The reductions in the case $H=0$ (i.e., $w=1$ ) are similar to those for the square-lattice strip, so we do not discuss them in detail.

\section{SOME THERMODYNAMIC PROPERTIES}

\section{A. Thermodynamic Functions}

For a lattice strip graph $G_{m}$ of length $L_{x}=m$ vertices with $n=n\left(G_{m}\right)$ vertices, we denote the formal limit of infinite length as

$$
\lim _{m \rightarrow \infty} G_{m} \equiv\{G\}
$$

The corresponding dimensionless reduced free energy $f$ in the limit $m \rightarrow \infty$ and hence $n=m L_{y} \rightarrow \infty$ as

$$
f(\{G\}, q, s, v, w)=\lim _{n \rightarrow \infty} n^{-1} Z\left(G_{m}, q, s, v, w\right)
$$


so that the actual Gibbs free energy is $\mathcal{G}(T, H)=-k_{B} T f$. For the $G_{m}=\Lambda, 2 \times m$, cyc. cyclic strip graphs considered here, in the $m \rightarrow \infty$ limit, only the $\lambda_{Z, \Lambda, 2, d, j}$ of maximum magnitude contributes. This comes from the set of terms with $d=0$ [1, 2, 4, 9, 15]. This is in accord with the property that $f$ should be independent of the (longitudinal) boundary condition and the fact that only the $d=0$ sector is present for free longitudinal boundary conditions.

For the square-lattice ladder strip, among the respective $s^{2}+2 s+2$ terms with $d=0$ that contribute to Eq. (3.1), we find that the dominant one is the term of largest magnitude among the five roots of the characteristic polynomial of the transfer matrix $T_{Z, s q, 2,0}$ given in Appendix B. For the triangular-lattice ladder strip, among the respective $s^{2}+2 s+2$ terms with $d=0$ that contribute to Eq. (3.1), we find that the dominant one is the term of largest magnitude among the six roots of the characteristic polynomial of the transfer matrix $T_{Z, t r i, 2,0}$ (see Appendix $\mathrm{C}$ ).

Thermodynamic functions are obtained from the reduced free energy (6.2) in the usual way. For first derivatives, the internal energy per vertex is $U=-\partial f / \partial \beta=-J y \partial f / \partial y$ and a magnetization per vertex

$$
M=\frac{\partial f}{\partial h}=w \frac{\partial f}{\partial w} .
$$

It is conventional to define a corresponding magnetic order parameter per vertex as (e.g., [7])

$$
\mathcal{M}=\frac{q M-1}{q-1}
$$

In the zero-field case, with this definition, in the high-temperature phase, where the global $S_{q}$-symmetry is realized manifestly, the spins take on random values in the set $I_{q}$, so $M=1 / q$ and hence $\mathcal{M}=0$. In the case of complete ferromagnetic ordering, $M=1$, and hence also $\mathcal{M}=1$.

\section{B. Cases where Frustration and Competing Interactions Occur}

One of the interesting features of our results is that they provide exactly solved examples that exhibit the occurrence of frustration and competing interactions as a function of a parameter, $s$, that one can choose. As discussed in the introduction, in the antiferromagnetic case with $J<0$, if $H>0$ (i.e., $w>1$ ) and $s<\chi(G)$ or if $H<0$ (i.e., $0 \leq w<1$ ) and $q-s<\chi(G)$, then the imposition of the respective positive or negative $H$ involves competing interactions and causes frustration. Given the symmetry (2.12), without loss of generality, it will suffice to discuss only the case of $H>0$, and we shall do so. For our lattice strip 
graphs,

$$
\begin{gathered}
\chi\left(s q, L_{y} \times m, c y c .\right)= \begin{cases}2 & \text { if } m \text { is even } \\
3 & \text { if } m \text { is odd }\end{cases} \\
\chi\left(\text { tri } L_{y} \times m, c y c .\right)=\left\{\begin{array}{ll}
3 & \text { if } m=0 \bmod 3 \\
4 & \text { if } m=1 \text { or } m=2 \bmod 3
\end{array} .\right.
\end{gathered}
$$

Since the limit $m \rightarrow \infty$ can be taken with even $m$ values for the cyclic square-lattice strip and with values of $m$ that are equal to $0 \bmod 3$ for the triangular-lattice strip, and since only the dominant $\lambda_{Z, \Lambda, L_{y}, 0, j}$ is relevant in this limit, it follows that the competition and frustration is exhibited in this dominant $\lambda_{Z, \Lambda, L_{y}, 0, j}$ for $H>0$ and $0<s<2$, i.e., $s=1$ for cyclic square-lattice strips and $0<s<3$, i.e., $s=1$ or $s=2$, for cyclic triangular-lattice strips. In each case, in accordance with our discussion in the introduction, it is assumed that in these respective cases, the chromatic number ( 2 or 3 ) is no greater than $q$, so that the trivial situation where $s=q$ does not occur. Similarly, there is frustration if $H<0$ and $0<q-s<2$ for square-lattice strips and $0<q-s<3$ for triangular-lattice strips.

Our discussion above is general. An explicit example of this phenomenon is instructive. The simplest illustration of the frustration is provided by $Z$ for the circuit graph, $Z\left(C_{m}, q, s, v, w\right)$. Here, the dominant term is $\lambda_{Z, C, 0,+}$ (given in Eqs. (3.19)-(3.21) $)$, so

$$
f(\{C\}, q, v, s, w)=\ln \left[\lambda_{Z, C, 0,+}\right]
$$

where, as in Eq. (6.1), $\mathcal{C}$ denotes the formal limit of the circuit graph $C_{m}$ as $m \rightarrow \infty$. For our discussion, it will be useful to have the explicit form of $M$ resulting from Eq. (6.7). We calculate

$$
M=\frac{w}{\sqrt{R}}\left[s+v-\frac{2 v(q+v)}{A+\sqrt{R}}\right],
$$

where $A$ and $R$ were given in Eqs. (3.20) and (3.21). The magnetic order parameter is then obtained by substituting this expression for $M$ in Eq. (6.4).

In the limit $m \rightarrow \infty$, taken on even $m, \chi(\{C\})=2$. If $J<0$ with $H>0$, then as $T \rightarrow 0$, the spin-spin interaction requires that the spins on adjacent vertices should be different, while the magnetic field term biases them to lie in the interval $I_{s}$. These requirements conflict with each other if $s=1$, since this is less than $\chi(\{C\})$. In this $s=1$ case, as $T \rightarrow 0$,

$$
\lambda_{Z, C, 0,+} \rightarrow \frac{1}{2}\left[q-2+e^{h-|K|}+\sqrt{\left(q-2+e^{h-|K|}\right)^{2}+4(q-1) e^{h}}\right]
$$

(where we have used the fact that $v \rightarrow-1$ in this limit). Now, if $H /|J|<1$, i.e., $h /|K|<1$, then $e^{h-|K|} \rightarrow 0$ as $T \rightarrow 0$, while $e^{h}$ grows without bound, so $\lambda_{Z, C, 0,+} \rightarrow \sqrt{(q-1) e^{h}}$ asymptotically, up to exponentially smaller correction terms. If $1<h /|K|<2$, then, although the $e^{h-|K|}$ term outside the square root and the $e^{2(h-|K|)}$ term inside the square root 
grow, they grow less rapidly than the $4(q-1) e^{h}$ term inside the square root, so as $T \rightarrow 0$, the dominant term in $\lambda_{Z, C, 0,+}$ asymptotically is again $\sqrt{(q-1) e^{h}}$. If $h /|K|>2$, then the $e^{2(h-|K|)}$ term inside the square root dominates over the $4(q-1) e^{h}$ term so, including also the $e^{h-|K|}$ term outside the square root, one finds that, as $T \rightarrow 0, \lambda_{Z, C, 0,+}$ asymptotically approaches $e^{h-|K|}$, with exponentially smaller correction terms. These are the generic intervals in $h /|K|$. One can also trace this demarcation point $h /|K|=2$ separating the two types of behavior in the space of complexified parameters, as, e.g., in another exactly solved spin model exhibiting frustration and competing interactions [16]. For completeness, one should also note the marginal case where $H=2|J|$, i.e., $h /|K|=2$, which forms a set of measure zero in the space of the parameters $J$ and $H$; in this case, if one expresses $\lambda_{Z, C, 0,+}$ in terms of $|K|$ alone, then as $T \rightarrow 0, \lambda_{Z, C, 0,+} \rightarrow(1 / 2) e^{|K|}[1+\sqrt{4 q-3}]$.

We now apply this analysis to $M$, as calculated in Eq. (6.8) and the resultant $\mathcal{M}$ from Eq. (6.4). In the case that we are considering, with $J<0$ and $s=1$, as $T \rightarrow 0$, if $H>2|J|$, the ferromagnetic tendency due to the external field dominates over the proper $q$-coloring tendency due to the spin-spin interaction, so

$$
J<1, H>2|J|, s=1 \Rightarrow M=\mathcal{M}=1 .
$$

If, on the other hand, $H<2|J|$, then

$$
J<1, H<2|J|, s=1 \Rightarrow M=\frac{1}{2}, \quad \mathcal{M}=\frac{q-2}{2(q-1)} .
$$

Thus, for $J<0$ and $s=1$, in the limit $T \rightarrow 0$, as $H$ decreases through the value $2|J|$, there is a discontinuous decrease in $\mathcal{M}$ from 1 to the value in Eq. (6.11). For $q=2$, this latter value is 0 ; for $q=3$, it is $1 / 4$, and as $q \rightarrow \infty$, it approaches $1 / 2$ from below. In this situation with frustration and competing interactions, there is thus a nonanalytic change in the magnetization at $T=0$ as a consequence of a change in the ratio $H /|J|$. Our example has the appeal that the physics is manifestly evident because of the explicit exact solution.

\section{CONCLUSIONS}

In this paper we have presented an exact calculations of the partition functions $Z(\Lambda, 2 \times$ $m ; q, s, v, w)$ of the $q$-state Potts model in a generalized magnetic field, on cyclic ladder strip graphs of the square and triangular lattices of arbitrary length. Several interesting analytic properties of these results have been elucidated. In particular, we have shown that, for the case of antiferromagnet spin-spin coupling, these provide exactly solved models that exhibit an onset of frustration and competing interactions, with a resultant nonanalytic change in 
physical quantities at zero temperature, in the context of a novel type of tensor-product $S_{s} \otimes S_{q-s}$ global symmetry.

\section{Acknowledgments}

This research was partly supported by the Taiwan Ministry of Science and Technology grant MOST 103-2918-I-006-016 (S.-C.C.) and by the U.S. National Science Foundation grant No. NSF-PHY-13-16617 (R.S.).

\section{Appendix A: $Z(s q, 2 \times m, c y c ., q, v)$}

We review here the result for the partition function $Z(s q, 2 \times m, c y c ., q, v)$ of the cyclic square-lattice ladder graph of length $L_{x}=m$ vertices presented in [1]. We include this in connection with our discussion in the text showing how our new result for $Z(s q, 2 \times$ $m, c y c ., q, s, v, w)$ reduces to $Z(s q, 2 \times m, c y c ., q, v)$ in the zero-field case $w=1$. This partition function has the form of

$$
Z\left(s q, L_{y} \times m, c y c ., q, v\right)=\sum_{d=0}^{L_{y}} c^{(d)} \sum_{j=1}^{n_{Z}\left(L_{y}, d\right)}\left(\lambda_{s q, L_{y}, d, j}\right)^{m}
$$

with $L_{y}=2$, where $n_{Z}(2,0)=2, n_{Z}(2,1)=3, n_{Z}(2,2)=1$, and the coefficients $c^{(d)}$ are given in Eq. (3.2), so

$$
c^{(0)}=1, \quad c^{(1)}=q-1, \quad c^{(2)}=q^{2}-3 q+1 .
$$

As is evident in Eq. (A1), to distinguish the $\lambda_{s q, 2, d, j} \mathrm{~s}$ in the zero-field partition function $Z(s q, 2 \times m, q, v)$ from the $\lambda_{Z, s q, 2, d, j}$ in the field-dependent partition function $Z(s q, 2 \times$ $m, q, s, v, w)$, we suppress the subscript $Z$ in the former. Explicitly,

$$
\begin{gathered}
Z(s q, 2 \times m, c y c ., q, v)=\left(\lambda_{s q, 2,0,+}\right)^{m}+\left(\lambda_{s q, 2,0,-}\right)^{m} \\
+c^{(1)}\left[\left(\lambda_{s q, 2,1,1}\right)^{m}+\left(\lambda_{s q, 2,1,+}\right)^{m}+\left(\lambda_{s q, 2,1,-}\right)^{m}\right]+c^{(2)}\left(\lambda_{s q, 2,2}\right)^{m},
\end{gathered}
$$

where (in order of decreasing $d$ ) $\lambda_{s q, 2,2}=v^{2}$,

$$
\lambda_{s q, 2,1,1}=v(q+v),
$$

$$
\lambda_{s q, 2,1,(2,3)} \equiv \lambda_{s q, 2,1, \pm}
$$




$$
=\frac{v}{2}\left[q+v(v+4) \pm\left(v^{4}+4 v^{3}+12 v^{2}-2 q v^{2}+4 q v+q^{2}\right)^{1 / 2}\right]
$$

and

$$
\lambda_{s q, 2,0,(1,2)} \equiv \lambda_{s q, 2,0, \pm}=\frac{1}{2}\left(A_{s q d 0} \pm \sqrt{R_{s q d 0}}\right)
$$

where

$$
A_{s q d 0}=v^{3}+4 v^{2}+3 q v+q^{2}
$$

and

$$
R_{s q d 0}=v^{6}+4 v^{5}-2 q v^{4}-2 q^{2} v^{3}+12 v^{4}+16 q v^{3}+13 q^{2} v^{2}+6 q^{3} v+q^{4} .
$$

The reader is referred to [2] for our corresponding solution for the partition function $Z$ (tri, $2 \times$ $m, c y c ., q, v)$ of the cyclic triangular-lattice ladder graph of arbitrary length.

\section{Appendix B: $T_{Z, s q, 2,0}$}

Five of the $s^{2}+2 s+2 \lambda_{Z, s q, 2,0, j}$ terms, each with multiplicity 1, are determined as the roots of a quintic equation which is the characteristic polynomial of the transfer matrix $T_{Z, s q, L_{y}, d}$ with $L_{y}=2, d=0$, and the following entries:

$$
\begin{gathered}
\left(T_{Z, s q, 2,0}\right)_{1,1}=\tilde{q}^{2}+3 v(\tilde{q}+v) \\
\left(T_{Z, s q, 2,0}\right)_{1,2}=2 s(\tilde{q}+v) w \\
\left(T_{Z, s q, 2,0}\right)_{1,3}=s(v+1) w^{2} \\
\left(T_{Z, s q, 2,0}\right)_{1,4}=s(s-1) w^{2} \\
\left(T_{Z, s q, 2,0}\right)_{1,5}=(\tilde{q}+2 v)(v+1) \\
\left(T_{Z, s q, 2,0}\right)_{2,1}=\tilde{q}^{2}+v(2 \tilde{q}+v) \\
\left(T_{Z, s q, 2,0}\right)_{2,2}=w[2 s \tilde{q}+v(q+v)] \\
\left(T_{Z, s q, 2,0}\right)_{2,3}=(v+1)(v+s) w^{2} \\
\left(T_{Z, s q, 2,0}\right)_{2,4}=(v+s)(s-1) w^{2} \\
\left(T_{Z, s q, 2,0}\right)_{2,5}=(\tilde{q}+v)(v+1) \\
\left(T_{Z, s q, 2,0}\right)_{3,1}=\left(T_{Z, s q, 2,0}\right)_{4,1}=\tilde{q}(\tilde{q}+v) \\
\left(T_{Z, s q, 2,0}\right)_{3,2}=\left(T_{Z, s q, 2,0}\right)_{4,2}=2 \tilde{q}(v+s) w \\
\left(T_{Z, s q, 2,0}\right)_{3,3}=(v+1)\left(v^{2}+2 v+s\right) w^{2}
\end{gathered}
$$




$$
\begin{gathered}
\left(T_{Z, s q, 2,0}\right)_{3,4}=(2 v+s)(s-1) w^{2} \\
\left(T_{Z, s q, 2,0}\right)_{3,5}=\left(T_{Z, s q, 2,0}\right)_{4,5}=\tilde{q}(v+1) \\
\left(T_{Z, s q, 2,0}\right)_{4,3}=(v+1)(2 v+s) w^{2} \\
\left(T_{Z, s q, 2,0}\right)_{4,4}=\left[(s+v)^{2}-s-2 v\right] w^{2} \\
\left(T_{Z, s q, 2,0}\right)_{5,1}=v^{3} \\
\left(T_{Z, s q, 2,0}\right)_{5, j}=0 \quad \text { for } 2 \leq j \leq 4 \\
\left(T_{Z, s q, 2,0}\right)_{5,5}=v^{2}(v+1)
\end{gathered}
$$

\section{Appendix C: Matrices $T_{Z, t r i, 2, d}$ for $d=1, d=0$}

1. $d=1$

Five of the $s^{2}+2 s+2 \lambda_{Z, t r i, 2,1, j}$ terms, each with multiplicity 1, are determined as the roots of a quintic equation which is the characteristic polynomial of the matrix $T_{Z, t r i, L_{y}, d}$ with $L_{y}=2, d=1$, and the following entries:

$$
\begin{gathered}
\left(T_{Z, t r i, 2,1}\right)_{1,1}=v \tilde{q}+2 v^{2} \\
\left(T_{Z, t r i, 2,1}\right)_{1,2}=s w v \\
\left(T_{Z, t r i, 2,1}\right)_{1,3}=\left(T_{Z, t r i, 2,1}\right)_{2,3}=v^{2} \\
\left(T_{Z, t r i, 2,1}\right)_{1,4}=\left(T_{Z, t r i, 2,1}\right)_{2,4}=0 \\
\left(T_{Z, t r i, 2,1}\right)_{1,5}=\left(T_{Z, t r i, 2,1}\right)_{2,5}=v(1+v) \\
\left(T_{Z, t r i, 2,1}\right)_{2,1}=v \tilde{q}+v^{2} \\
\left(T_{Z, t r i, 2,1}\right)_{2,2}=w v(v+s) \\
\left(T_{Z, t r i, 2,1}\right)_{3,1}=\left(T_{Z, t r i, 2,1}\right)_{4,1}=v \tilde{q}+3 v^{2}+v^{3} \\
\left(T_{Z, t r i, 2,1}\right)_{3,2}=\left(T_{Z, t r i, 2,1}\right)_{4,2}=s w v \\
\left(T_{Z, t r i, 2,1}\right)_{3,3}=v \tilde{q}+4 v^{2}+v^{3} \\
\left(T_{Z, t r i, 2,1}\right)_{3,4}=s w v \\
\left(T_{Z, t r i, 2,1}\right)_{3,5}=\left(T_{Z, t r i, 2,1}\right)_{4,5}=v(1+v)(2+v) \\
\left(T_{Z, t r i, 2,1}\right)_{4,3}=v \tilde{q}+3 v^{2}+v^{3}
\end{gathered}
$$




$$
\begin{gathered}
\left(T_{Z, t r i, 2,1}\right)_{4,4}=w v(v+s) \\
\left(T_{Z, t r i, 2,1}\right)_{5,1}=v^{2} \tilde{q}+3 v^{3}+v^{4} \\
\left(T_{Z, t r i, 2,1}\right)_{5,2}=s w v^{2} \\
\left(T_{Z, t r i, 2,1}\right)_{5,3}=3 v^{3}+v^{4} \\
\left(T_{Z, t r i, 2,1}\right)_{5,4}=0 \\
\left(T_{Z, t r i, 2,1}\right)_{5,5}=v^{2}(1+v)(2+v)
\end{gathered}
$$

\section{2. $d=0$}

Five of the $\lambda_{Z, t r i, L_{y}, d, j}$ with $L_{y}=2$ and $d=0$ are the roots, each with multiplicity $s-1$, of a quintic equation which is the characteristic polynomial of the transfer matrix $T_{Z, t r i, 2,0 a}$. This matrix may be obtained from $T_{Z, t r i, 2,1}$ by the replacements $s \rightarrow q-s$ and $w \rightarrow w^{-1}$ and then multiplication by $w^{2}$.

Six of the $\lambda_{Z, t r i, L_{y}, d, j}$ with $L_{y}=2$ and $d=0$ are the roots, each with multiplicity 1 , of a degree- 6 equation which is the characteristic polynomial of the matrix $T_{Z, t r i, 2,0 b}$ with entries

$$
\begin{gathered}
\left(T_{Z, t r i, 2,0 b}\right)_{1,1}=\tilde{q}^{2}+4 v \tilde{q}+5 v^{2}+v^{3} \\
\left(T_{Z, t r i, 2,0 b}\right)_{1,2}=s w(\tilde{q}+v) \\
\left(T_{Z, t r i, 2,0 b}\right)_{1,3}=s w(\tilde{q}+2 v) \\
\left(T_{Z, t r i, 2,0 b}\right)_{1,4}=s w^{2}(1+v) \\
\left(T_{Z, t r i, 2,0 b}\right)_{1,5}=s(s-1) w^{2} \\
\left(T_{Z, t r i, 2,0 b}\right)_{1,6}=(1+v)\left(\tilde{q}+3 v+v^{2}\right) \\
\left(T_{Z, t r i, 2,0 b}\right)_{2,1}=\tilde{q}^{2}+3 v \tilde{q}+3 v^{2}+v^{3} \\
\left(T_{Z, t r i, 2,0 b}\right)_{2,2}=w(v+s)(\tilde{q}+v) \\
\left(T_{Z, t r i, 2,0 b}\right)_{2,3}=s w(\tilde{q}+v) \\
\left(T_{Z, t r i, 2,0 b}\right)_{2,4}=w^{2}(1+v)(v+s) \\
\left(T_{Z, t r i, 2,0 b}\right)_{2,5}=w^{2}(s-1)(v+s) \\
\left(T_{Z, t r i, 2,0 b}\right)_{2,6}=(1+v)\left(\tilde{q}+2 v+v^{2}\right) \\
\left(T_{Z, t r i, 2,0 b}\right)_{3,1}=\tilde{q}^{2}+2 v \tilde{q}+v^{2}
\end{gathered}
$$




$$
\begin{aligned}
& \left(T_{Z, t r i, 2,0 b}\right)_{3,2}=w \tilde{q}(v+s) \\
& \left(T_{Z, t r i, 2,0 b}\right)_{3,3}=w(v+s)(\tilde{q}+v) \\
& \left(T_{Z, t r i, 2,0 b}\right)_{3,4}=w^{2}(1+v)\left(s+2 v+v^{2}\right) \\
& \left(T_{Z, t r i, 2,0 b}\right)_{3,5}=w^{2}\left(2 v s-2 v-s+s^{2}\right) \\
& \left(T_{Z, t r i, 2,0 b}\right)_{3,6}=(1+v)(\tilde{q}+v) \\
& \left(T_{Z, t r i, 2,0 b}\right)_{4,1}=\left(T_{Z, t r i, 2,0 b}\right)_{5,1}=\tilde{q}(\tilde{q}+v) \\
& \left(T_{Z, t r i, 2,0 b}\right)_{4,2}=w \tilde{q}\left(s+2 v+v^{2}\right) \\
& \left(T_{Z, t r i, 2,0 b}\right)_{4,3}=\left(T_{Z, t r i, 2,0 b}\right)_{5,3}=w \tilde{q}(s+v) \\
& \left(T_{Z, t r i, 2,0 b}\right)_{4,4}=w^{2}(1+v)\left(s+3 v+3 v^{2}+v^{3}\right) \\
& \left(T_{Z, t r i, 2,0 b}\right)_{4,5}=w^{2}(s-1)\left(s+3 v+v^{2}\right) \\
& \left(T_{Z, t r i, 2,0 b}\right)_{4,6}=\left(T_{Z, t r i, 2,0 b}\right)_{5,6}=\tilde{q}(1+v) \\
& \left(T_{Z, t r i, 2,0 b}\right)_{5,2}=w \tilde{q}(s+2 v) \\
& \left(T_{Z, t r i, 2,0 b}\right)_{5,4}=w^{2}(1+v)\left(s+3 v+v^{2}\right) \\
& \left(T_{Z, t r i, 2,0 b}\right)_{5,5}=w^{2}\left(s^{2}-s+3 v s-3 v+v^{2}\right) \\
& \left(T_{Z, t r i, 2,0 b}\right)_{6,1}=v^{2}\left(\tilde{q}+3 v+v^{2}\right) \\
& \left(T_{Z, t r i, 2,0 b}\right)_{6,2}=\left(T_{Z, t r i, 2,0 b}\right)_{6,4}=\left(T_{Z, t r i, 2,0 b}\right)_{6,5}=0 \\
& \left(T_{Z, t r i, 2,0 b}\right)_{6,3}=s w v^{2} \\
& \left(T_{Z, t r i, 2,0 b}\right)_{6,6}=v^{2}(1+v)(2+v)
\end{aligned}
$$

[1] Shrock, R.: Physica A 283, 388 (2000).

[2] Chang, S.-C. and Shrock, R.: Physica A 286, 189 (2000).

[3] Chang, S.-C. and Shrock, R.: J. Stat. Phys. 137, 667 (2009).

[4] Chang, S.-C., Shrock, R.: J. Stat. Phys., 138, 496 (2010).

[5] W. F. Wreszinski and S. R. A. Salinas, Disorder and Competition in Soluble Lattice Models (World Scientific, Singapore, 1993).

[6] Wu, F. Y.: J. Stat. Phys 18115 (1978). 
[7] Wu, F. Y.: Rev. Mod. Phys. 54, 235 (1982).

[8] Chang, S.-C., Shrock, R.: J. Phys. A 42, 385004 (2009).

[9] Shrock, R., Xu, Y.: J. Stat. Phys. 139, 27 (2010).

[10] Shrock, R., Xu, Y.: J. Stat. Phys 141, 909 (2010).

[11] Tutte, W. T.: Proc. Camb. Phil. Soc. 43, 26 (1947).

[12] Tutte, W. T.: J. Combin. Theory 1, 301 (1967).

[13] N. Biggs, Algebraic Graph Theory (Cambridge Univ. Press, Cambridge, 1993).

[14] B. Bollobás, Modern Graph Theory (Springer, New York, 1998).

[15] Chang, S.-C. and Shrock, R.: Physica A 296, 234 (2001).

[16] Shrock, R. and Tsai, S-H.: Phys. Rev. E 55, 5184 (1997). 